non-urgent problems are directed to general practitioners (GPs) and other primary care clinicians working within or alongside EDs to address increasing demand. Our findings describe variation in the ways that these primary care services are implemented and the ways in which GPs work within them. We also highlight successes and challenges in implementing such services.

Methods/Design We conducted interviews with ED clinical leads in England $(n=19)$ and Wales $(n=2)$. We used framework analysis to analyse interview transcripts and explore differences across 'primary care services', 'emergency medicine services' and emergency departments without primary care services.

Results/Conclusions In EDs with separate primary care services, success was reported with a distinct workforce of primary care clinicians, who improved waiting times and flow by seeing primary care-type patients in a timely way, using fewer investigations, and enabling ED doctors to focus on more acutely unwell patients. Some challenges were: aligning services with the policy guidance, inconsistent demand for primary care, accessible community primary care services, difficulties recruiting GPs, lack of funding, difficulties agreeing governance protocols and establishing effective streaming pathways. Where GPs were integrated into an ED workforce success was reported as managing the demand for both emergency and primary care and reducing admissions.

To support successful and sustainable primary care services in or alongside EDs, policy makers and commissioners should consider varied ways that GPs can be employed to manage local demand and also local contextual factors such as the ability to recruit and retain GPs, sustainable funding, clear governance frameworks, training, support and guidance for all staff.

\section{PATIENTS'MOTIVATIONS AND EXPECTATIONS WHEN SEEKING URGENT CARE AT EMERGENCY DEPARTMENTS AND ACCEPTABILITY OF PRIMARY CARE STREAMING: A REALIST STUDY}

'Michelle Edwards, 'Delyth Price, 'Julie Hepburn, 'Barbara Harrington, ${ }^{2}$ Bridie Evans, ${ }^{1}$ Alison Cooper, ${ }^{1}$ Adrian Edwards. ${ }^{1}$ Cardiff University; ${ }^{2}$ Swansea University

\subsection{6/emj-2020-rcemabstracts. 13}

Aims/Objectives/Background We aim to explain the contexts and mechanisms that influence patients' motivations and expectations when accessing urgent care at an ED and their acceptability of being streamed to a primary care clinician working in or alongside the ED. Recent healthcare policy has encouraged the implementation of primary care services in or alongside emergency departments whereby patients with low acuity illness are streamed to a primary care clinician after a brief initial assessment. Our findings describe patients' motivations, expectations, and acceptability of primary care streaming and their level of satisfaction.

Methods/Design We recruited 24 patients to be interviewed after visiting an emergency department for one of five low acuity complaints. 12 patients were streamed to ED clinicians and 11 were streamed to primary care clinicians. We carried out semi-structured realist style interviews by telephone and carried out a realist analysis to create theories to explain motivations to attend, acceptability of streaming and satisfaction with care.
Results/Conclusions Motivations for attending the ED included patients' perception of their complaint as an emergency which needed immediate treatment, and previous experience of receiving care at the ED. Acceptability of primary care streaming was related to patients' past experiences accessing primary care services, their trust in initial assessment processes and their expectation to be seen by 'expert clinicians' on the 'same day'. When patients' expectations of waiting times, level of investigations and general quality of care were met or exceeded, they reported acceptability to being streamed to a primary care clinician and were satisfied with their care. Understanding why patients attend the ED for urgent care needs and their experience of primary care streaming is essential to addressing increasing ED demand and improving efficiency.

\section{RCEM lightning}

\section{CAN NEWS2 BE USED TO PREDICT A\&E RE- ATTENDANCES DURING THE COVID-19 PANDEMIC?}

Krishna Prasad Gopalakrishna Pillai, Zeyar Win Naing, Hemavathi Muniswamy, Abdul Sajjad Pathan. Luton and Dunstable University Hospital

\subsection{6/emj-2020-rcemabstracts. 14}

Aims/Objectives/Background The NEWS2 (National Emergency Warning Score 2) is a widely used tool in Emergency Departments (ED) to identify patients who may be at increased risk of deterioration. NEWS2 is based on a simple aggregate scoring system in which a score is allocated to physiological measurements, already recorded in routine practice, when patients present to, or are being monitored in hospital. Currently there are no predictive scoring systems to evaluate ED re-attendances. The objective of this study was to investigate whether calculated NEWS2 had any influence on the ED re-attendances during the COVID-19 pandemic.

Methods/Design Data for the study was compiled from Symphony, the department's electronic patient records. Data was retrospectively compiled for the month of April 2020 and was subsequently followed up to 28 days for re-attendances. Patients aged 18 years and older, presenting with COVID-19 symptoms who were discharged from ED were only included $(n=310)$. Case definitions for COVID-19 symptomatology were in accordance to the Public Health England guidance.

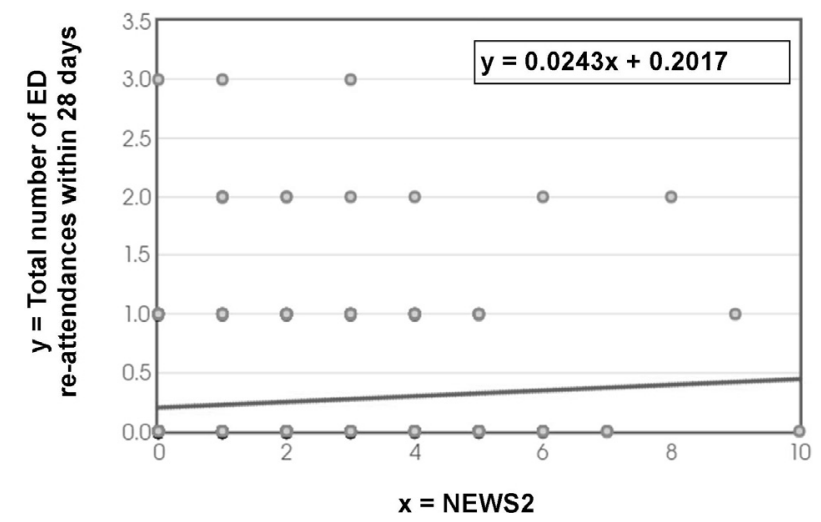

Abstract 301 Figure 1 
Correlation was made between NEWS2 and the total number of re-attendances within 28 days using regression analysis.

Results/Conclusions Simple linear regression analyses the influence of NEWS2 and the total number of re-attendances within 28 days and gives us the value of $\mathrm{R}^{2}$ as 0.007 , which suggests that 0.7 percent of variation of NEWS2 is attributable to regression (figure 1). The regression line is almost flat which tells us that there is no relationship between the two variables. NEWS2 alone is not a useful tool to predict reattendances to ED for patients presenting with COVID-19 symptoms.

\section{QUALITY IMPROVEMENT PROJECT - IMPROVING THE QUALITY OF CARE FOR HOMELESS PATIENTS PRESENTING TO THE HOMERTON UNIVERSITY HOSPITAL EMERGENCY DEPARTMENT 2019-20}

Phoebe Leung, Jane Chambers, Amber Morris, Kobbina Arthur, Fenella Prowse, Richard Crowson, William Niven. Homerton university hospital

\subsection{6/emj-2020-rcemabstracts. 15}

Aims/Objectives/Background Homelessness is on the rise in the UK. The problem identified specific to homeless patient care was clinician understanding of the homeless person's social needs to form an adequate discharge plan as well as completing their legal duty to refer such patients to the local housing authority.

Methods/Design This quality improvement project (QIP) aimed to reduce the reattendance rate of homeless patients presenting to the Homerton University Hospital (HUH) Emergency Department (ED) by 20\% from November 2019 to April 2020. This would be done by improving social history taking, signposting of patients to appropriate resources, and performing the legal duty to refer. Using the PDSA cycle method, interventions included a week of presentations to inform clinicians of the process measures; an advertising campaign; and a defined flowchart process for the duty to refer.

Results/Conclusions The QIP yielded the following results in terms of median baselines: social history taking $60 \%$ to $88 \%$, signposting to resources $30 \%$ to $67 \%$, and duty to refer 0 to $41 \%$. There was no change to the outcome measure of reattendance rate, maintained at $40 \%$ throughout the project and hence the QIP did not meet its SMART aim.

However this may have been the result of the decision to cut short data collection time due to the unprecedented COVID-19 pandemic which saw overall reduction in ED patient attendance. Most street homeless persons were put up in temporary hotels in the government funded scheme 'Everybody In', lockdown meant the hidden homeless should stay indoors, and a ban on court evictions has been extended until 23 August 2020.

Nonetheless, work to improve quality of care continued with a new pathway for safe discharge of homeless patients with suspected COVID-19. Planning ahead for post pandemic times has brought about a new standard operating procedure, which will ensure sustainability of the QIP.

\section{NHS 24 TRIAGE OF THE YOUNG ADULT WITH CHEST PAIN: AN ANALYSIS OF PATIENT CONTACTS IN UNSCHEDULED CARE}

${ }^{1}$ Peter Hodgins, ${ }^{2}$ Bruce Guthrie, ${ }^{2}$ Megan McMinn. ' Usher Institute, University of Edinburgh; ${ }^{2}$ University of Edinburgh

\subsection{6/emj-2020-rcemabstracts. 16}

Aims/Objectives/Background Use of unscheduled care is increasing worldwide. In the UK access to services for symptoms not thought to be life-threatening is through a single telephone advice and triage service (NHS 24 in Scotland). Adults under 35 account for the largest cohort contacting this

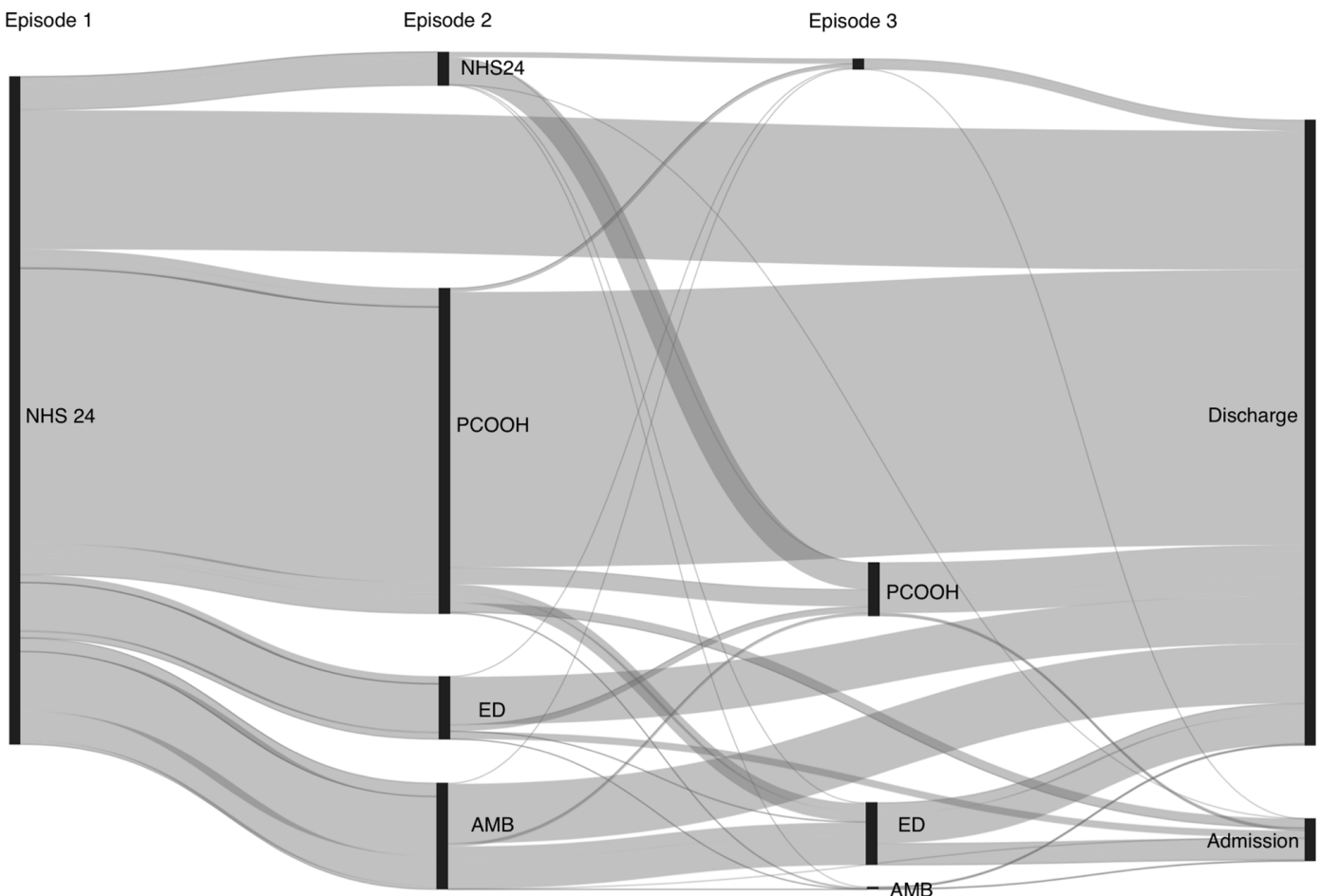

